Sciendo

On Bickle's failure to give a formal account of the location in the new-wave reductionist spectrum

\author{
João Fonseca \\ Universidade Nova de Lisboa
}

Disputatio Vol. 1, No. 17

November 2004

DOI: $10.2478 /$ disp-2004-0011

ISSN: 0873-626X

(C) 2004 Fonseca. Creative Commons Attribution-NonCommercial-NoDerivs 3.0 License 


\title{
Critical notice
}

\section{On Bickle's failure to give a formal account of the location in the new-wave reductionist spectrum ${ }^{1}$}

\author{
João Fonseca \\ New University of Lisbon
}

\begin{abstract}
In this paper I discuss John Bickle's attempt to provide a formal procedure to locate a certain reduction relation in the Hooker's and Churchland's New wave reductionist spectrum. Bickle's main motivation is to react against the 'Khunnian flavored,' internal-to-scientific-practice pragmatist solution endorsed by Patricia Churchland when faced with the lack of a formal and external way to identify a reduction in the spectrum. Bickle tries to solve this problem by reformulating Hooker's insights within a structuralist framework so establishing an external-toscientific-practice formal solution. I show that Bickle fails on his attempt and, as a consequence, we should adopt Patricia Churchland pragmatic stance regarding reductionist and ontological decisions.
\end{abstract}

\section{The problem}

On Psychoneural Reduction (Bikcle 1998) John Bickle claims that the Hooker/Churchland reductionist model (labeled New Wave reduction-

\footnotetext{
${ }^{1}$ This work was developed as part of the research project POCTI/FIL/3455/ 99. I would like to thank the Portuguese Foundation for Science and Technology which granted me a post-doc fellowship. I am also grateful to João Sáágua for his constant support and encouragement and to an anonymous referee for his or hers helpful comments.
}

Disputatio, Vol. I, No. 17, November 2004 
ism) suffers from an important incompleteness. The problem lies in the lack of a formal procedure in order to locate a certain reduction in the spectrum 'from retention to elimination' provided by that very same reductionist model. In consequence, claims Bickle, ontological conclusions drawn from reductionist relations are vindicated in a pure intuitive and (somehow) arbitrary way. As an example he quotes Patricia Churchland from Neurophilosophy when confronted with this problem. She says the following:

Determining when the fit [reduction relation] is close enough to claim identities between properties of the old [theory] and those of the new is not a matter for formal criteria....I do not think it matters very much that we establish criteria for determining when the reduced and reducing theories resemble each other sufficiently to herald identity of properties (Churchland 1986, 283).

That is, given two theories establishing a reduction relation between them, we cannot tell precisely where that reduction fits in the reductionist spectrum. We should, then, let scientific practice itself 'decide' what kind of ontological relation those two theories establish. But, turning to science practice proper to adjudge, we inevitable fall into pragmatic and (hence) arbitrary procedures. Dismissing formal criteria:

the decision is influenced by a variety of pragmatic and social considerations. The whim of the central investigators, the degree to which confusion will result from retention of the old terms, the desire to preserve or to break with past habits of thought, the related opportunities for publicizing the theory, cadging grants, and attracting disciples all enter into decisions concerning whether to claim identities and therewith retention or whether to make more radical claim of displacement. (Churchland 1986, 283, 284)

For Bickle 'we should not ignore the disastrous limitations of this attitude if we wish to draw ontological conclusions based on the nature of the intertheoretic-reduction relation' $(1998,54)$ and criticizes Churchland for the inevitable adoption of a mere pragmatic perspective concerning the ontological topics. He concludes that 'sounding at first so brash, radical and empirically motivated, Churchland's ontological eleminativism now appears to be little more than an episode in the sociology of science' $(1998,54)$. So, Bickle aims for a 
method that tells us what is the ontological moral in a given reduction relation in an non arbitrary way.

\section{Bickle's structuralist solution}

Bickle's way out to overpass this hindrance is to reformulate newwave reductionism within a structuralist framework. Generally, structuralists adopt the models of a theory as the fundamental notion in their analysis. A certain theory $T$ is conceived as the set of structures that constitute the models of that theory. An important distinction should be made between 'real' or 'actual' models of a theory (simplifying: $M(T)$ ) and mere 'potential' models of the same theory $\left(\mathrm{M}_{\mathrm{P}}(\mathrm{T})\right)$. The latter kind of models should be viewed as structures of which it 'makes sense' to ask whether they are actual models (Bickle 1998, 63). We should also consider the intended empirical applications of the theory $(\mathrm{I}(\mathrm{T})$ ) and, within this set, the subset of confirmed empirical applications of the theory $(\operatorname{Ic}(\mathrm{T}))$. Consequently, a certain theory $T$ is considered as an ordered triple $\left.<\mathrm{M}_{\mathrm{P}}(\mathrm{T}), \mathrm{M}(\mathrm{T}), \mathrm{I}(\mathrm{T})\right\rangle$ in which $\mathrm{M}(\mathrm{T}) \subset \mathrm{M}_{\mathrm{P}}(\mathrm{T}), \mathrm{I}(\mathrm{T}) \subset \mathrm{M}_{\mathrm{P}}(\mathrm{T})$, and $\mathrm{Ic}(\mathrm{T})=\mathrm{M}(\mathrm{T}) \cap \mathrm{I}(\mathrm{T})$.

In a reduction context we have two theories: the theory to be reduced $\left(T_{R}\right)$ and the theory the former is reducible to $\left(T_{B}\right)$. In the new-wave reductionist framework we deduce an analogue theory of $T_{R}$ from $T_{B}$ plus counterfactual assumptions $C_{R}$ (the theory $T *_{R}$ ) and not $T_{R}$ itself as in the classic Nagelian model. Reformulated in a structuralist way we have, then, two sets: $T_{B}$ and $T_{R}$, and also $T *_{R}$ as a subset of $T_{B}$. We devise a reduction relation $\rho$ between $T_{B}$ and $T_{R}$ defined as a set of ordered pairs where the first member of each pair belongs to the intersection between $T^{*}{ }_{R}, M\left(T_{B}\right)$ and $I\left(T_{B}\right)$ and the second to $\operatorname{Ic}\left(T_{R}\right)$ (that is, to the intersection between $M\left(T_{R}\right)$ and $I\left(T_{R}\right)$ ). Thus, $\operatorname{Dom}(\rho)=\mathrm{T}^{*}{ }_{\mathrm{R}} \cap \mathrm{M}\left(\mathrm{T}_{\mathrm{B}}\right) \cap \mathrm{I}\left(\mathrm{T}_{\mathrm{B}}\right)$ and $\operatorname{Rng}(\rho)=\mathrm{M}\left(\mathrm{T}_{\mathrm{R}}\right) \cap \mathrm{I}\left(\mathrm{T}_{\mathrm{R}}\right)$.

Nevertheless, on many cases (including factual historical ones) the intersection between $T^{*}{ }_{R}$ with $\mathrm{M}\left(\mathrm{T}_{\mathrm{B}}\right)$ and $\mathrm{I}\left(\mathrm{T}_{\mathrm{B}}\right)$, and the intersection between $M\left(T_{R}\right)$ and $I\left(T_{R}\right)$ does not obtain (at all or to a certain 'pertinent' degree) given the counterfactual assumptions $C_{R}$ used in order

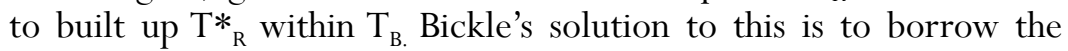
theoretical notion of 'blur' proposed by Balzer, Moulines and Sneed (1987) in order to immunize a theory on its empirical applications. Blurs are formal operations that take advantage of the potential models of a theory. They are members of an uniformity on the potential 
models of a theory imposing 'a topology on an otherwise unstructured set' (Bickle 1998, 84). More precisely, uniformities are sets of sets of ordered pairs of potential models. A given blur, being an element of an uniformity, is a set of ordered pairs of potential models of a theory. Changing the context from intra-theoretic immunization to inter-theoretic reduction Bickle uses blurs in order to deal with the 'intersection problem'. In a nutshell, Bickle's idea is to blur (via potential models) $M\left(T_{R}\right)$ (in symbols: ${ }^{\sim} M\left(T_{R}\right)$ ) into $I\left(T_{R}\right)$ in such a way that we obtain a 'significant' intersection between the two sets, and similarly with $\mathrm{M}\left(\mathrm{T}_{\mathrm{B}}\right)$ and $\mathrm{I}\left(\mathrm{T}_{\mathrm{B}}\right)\left({ }^{\sim} \mathrm{M}\left(\mathrm{T}_{\mathrm{B}}\right)\right.$ and $\left.{ }^{\sim} \mathrm{I}\left(\mathrm{T}_{\mathrm{B}}\right)\right)$ in relation to

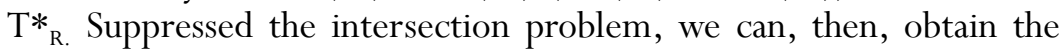
desired reduction relation between $T_{R}$ and $T_{B}$. Now, Bickle's upshot to overpass the problem of the localization in the reductive spectrum is to measure the blurs of each reduction relation, more specifically:

Being elements of uniformities, and hence sets of ordered pairs, each blur will have a cardinality determined by its bound. Bigger blurs have greater cardinality. They contain more ordered pairs. This feature provides a natural measure of smoothness of a reduction. Smoother reductions require smoother blurs on $\mathrm{M}\left(\mathrm{T}_{\mathrm{R}}\right), \mathrm{M}\left(\mathrm{T}_{\mathrm{B}}\right)$ and $\mathrm{I}\left(\mathrm{T}_{\mathrm{B}}\right)[\ldots]$ Blurs, at least partly, quantitatively capture our intuitions of approximation. Since their cardinality is the central component of this measure, approximative reductions can be ranked on a scale of the cardinality of the blurs. [...] Location on the intertheoretic reduction spectrum seems potentially amendable to quantitative analysis after all. (Bickle 1998, 97, 98)

This is, very concisely, Bickle's structuralist solution to the incompleteness of the new wave reductionist model.

\section{Bickle’s failure}

Although he does not put things directly this way, it is obvious that if Bickle is to succeed he must provide an effective procedure that tells us, given an arbitrary reduction $R$, what is its precise place on the intertheoretic reduction spectrum. To achieve this effective procedure one needs to satisfy (at least) the following conditions:

Condition 1: Blurs are finite and denumerable (sets of ordered pairs).

Condition 2: The cardinality of a given reduction $R$ corresponds to the sum of the cardinality of the three blurs associated with it. That is (more 
formally): $|R|=\sum\left(\left|\sim \mathrm{M}\left(\mathrm{T}_{\mathrm{R}}\right)\right| ; \quad{ }^{\sim} \mathrm{I}\left(\mathrm{T}_{\mathrm{B}}\right)|;|{ }^{\sim} \mathrm{M}\left(\mathrm{T}_{\mathrm{B}}\right) \mid\right)$. Where $|\mathrm{A}|$ stands for 'the cardinality of A'.

Condition 3: The values of the cardinality attached to the reductions range from 0 to $k$ (in the natural numbers), where 0 stands for the case when the reduction corresponds to the perfect retention and $k$ to the total replacement case. This defines the finite set of natural numbers $\mathrm{C}$.

Condition 4: The reductive spectrum is not a continuum in a mathematical sense but a finite set of natural numbers.

Condition 5: The values attached to the spectrum range from 0 to $n$ (in the natural numbers) where 0 stands for the perfect retention and $n$ for total replacement. This defines the finite set of natural numbers $\mathrm{S}$.

Condition 6: There is a recursive/computable function $\left(f_{\mathrm{R}}\right)$ establishing a one-to-one mapping between the range of the cardinality of the reductions (the set $\mathrm{C}$ ) and the domain of the values identifying a place in the reductionist spectrum (the set $\mathrm{S}$ ). The function should be such that for argument 0 it obtains the value 0 and for argument $k$ it obtains the value $n$ (obviously, the value of $n$ depends on the value we establish for $k$ ).

For the sake of the argument I will take the majority of the conditions for granted (for instance, I will not question the veracity of conditions 1 or 4). Condition 6 is crucial and I should clarify it.

The question is whether or not we have an algorithm in order to correspond to condition 6 . We have two possible scenarios. In the first one the three blurs grow in a proportional way in each case (recall condition 2). In this case we always have an algorithm and, consequently, grant that $f_{\mathrm{R}}$ is recursive. Take the simplest illustrative example: suppose that the blurs grow adding one member each (one ordered pair). Then, given condition 2, we would have the range of the cardinality of the reductions (the set $\mathrm{C}$ ) as a set of multiples of three: $\mathrm{C}=\{0,3,6,9, \ldots, k\}$. In order to obtain an one-to-one mapping between the members of $\mathrm{C}$ and $\mathrm{S}$ (where $\mathrm{S}=\{0,1,2,3, \ldots, n\}$ ), the function $f_{\mathrm{R}}(x)=y$ would be expressible by the simple algorithm: $y=$ $x / 3$, defining a set of ordered pairs: $\{<0,0>,<3,1>,<6,2>, \ldots$, $<k, n>\}$.

If the blurs do not allow us a proportionality and, in general, if we cannot write down an algorithm, $f_{\mathrm{R}}$ can, nevertheless still recursive/computable in the way demanded by condition 6 . The simple 
solution is to consider the function as an identity function: $y=x$. But there is the difficulty posed by the fact that, depending on the possible cases (on what the elements of the set $\mathrm{C}$ would be), that identity function could not be one-to-one (when sets $\mathrm{C}$ and $\mathrm{S}$ are not isomorphic) and possibly non-linear. In order to suppress this hindrance, I suggest the assumption of two different kinds of reductions: actual cases of reductions ('real' reductions) and 'virtual' reductions. 'Real' reductions would correspond to the cases where the cardinality of the blurs would count 'in fact' as reductions in a particular situation. On the other hand, 'virtual' reductions would, on the contrary, correspond to the cases where the cardinality of the blurs would not count as reductions at all in a particular situation. In this scenario, all cardinality would correspond to a reduction, real or virtual. So, we can grant an isomorphism between the sets $\mathrm{C}$ and $\mathrm{S}$. Thus, the identity function would define a set of ordered pairs where the first member of some of them will correspond to real and others to virtual reductions: $\{\langle 0,0\rangle,\langle 1,1\rangle,\langle 2,2\rangle, \ldots,\langle k, n\rangle\}$ (the 'extreme cases', $\langle 0,0\rangle$ and $\langle k, n\rangle$, would have to correspond to 'real' reductions). What matters is that given the cardinality of a certain (real) reduction we can obtain its value corresponding to the place in the reductionist spectrum. In fact, we can expand this (somewhat artificial) distinction between 'real' and 'virtual' reductions to use the identity function even when there is an algorithm available. So, the moral is that, in fact, we can always (in all possible circumstances) deploy a recursive function as demanded by condition 6 .

So, where is the trouble? The problem lies on how to establish a value for $k$. What is the cardinality of a reduction corresponding to the total replacement situation? Of course we can naturally attribute the cardinality 0 to the perfect retention situation (as stated in condition 3 ). But what about the other extreme? What is the bound value corresponding to the maximum blurs admissible? Bickle does not give a clue about this. In fact, it seems improbable to provide a mathematical procedure to decide this. Take the basic notion of 'potential models' (those needed to built up blurs). We would have to assume that all the theories have the same number of potential models. But such an assumption is far from obvious. We should take in consideration that in this structuralist framework, 'models are not representations of things depicted by a theory. Instead, they are the things depicted' (Bickle 1998, 62). Given this, depending on the nature of the theory (its 
constraints, scope and empirical domain) we would have different numbers of potential models for different theories, affecting the cardinality of the admissible blurs on each case (think, for instance, in a macro-physical theory compared to a micro-physical one).

Bickle himself recognizes (somehow) explicitly this difficulty when confronting the problem of distinguishing bumpy reductions from mere historical successions (those cases so radical that lie outside the reductionist spectrum). He says:

It is ridiculously optimistic to hope that we find some bound value on the cardinality of the required blurs separating blurs small enough to count as genuine reduction from blurs big enough to count as mere historical theory succession (1998, 100-101).

We could think that the solution would be to take an actual reduction corresponding to the total replacement situation and then check out the cardinality of the blurs required for that reduction. But this solution would be question begging, since we aim a method that tells us in a precise way where a particular reduction is to be placed in the spectrum, in order to correct our intuitive, non-formal way to do that.

At best, all we can tell is (assuming the same number of members of potential models for each theory) given some particular reductions $R$, $R^{\prime}$ and $R^{\prime \prime}$ that (for instance) $|R|>\left|R^{\prime}\right|>\left|R^{\prime \prime}\right|$ and establish that $R$ should be placed closer to the bumpier/elimination endpoint of the spectrum than $R^{\prime}$ and $R^{\prime \prime}$. Bickle seems, at times, to adopt such a mitigated and careful attempt. Taking as an example the reduction of physical optics to Maxwell's electrodynamics, he states that 'we need to include in the blur capturing them only a small number of ordered pairs, at least compared to the blurs required to get thermodynamics to reduce to kinetic theory' (1998, 97, emphasis added). So, Bickle vacillates, but this strategy is not a solution for our problem. We cannot tell in an external precise way what place in the spectrum a given reduction corresponds to. The same question begging problem arises: depending on the value we arbitrarily establish for $k$ we can, in fact, furnish such a location. But it would be a relative and parochial location and not the required precise one. 
We should not take this retraction as consisting in a middle ground success for Bickle. Although he stresses that 'I only intend this discussion to show that I can make quantitative sense of "amount of correction" that locates reductions on the smooth-to-bumpy spectrum' $(1998,101)$, his original attempt is clearly to provide a 'precise account of a given reduction's location on the spectrum' (1998, 74, emphasis added) in order to suppress the new-wave incompleteness. Bickle's structuralist solution to this hindrance clearly collapses.

\section{Morals}

Bickle somehow tends to establish independent and normative criteria over scientific activity proper. This is well illustrated in his motivation to correct the intuitive, non-formal arbitrary way by which we locate a certain reduction in the new-wave spectrum. Concerning ontological questions, Bickle wishes an external criteria in order to avoid the consequences he criticizes on Patricia Churchland and so, to escape the inevitability of falling on a mere 'episode in the sociology of science'.

Curiously enough, Bickle himself seems to assume that, contrarily to the classical/Nagelian model (under the ambition/propose of the 'unity of science'), the new-wave reductionist perspective does not impose regulative and normative constraints over 'normal' scientific development and activity. Bickle comments the anti-reductionists criticisms held by Fodor, Pylyshyn and Horgan, according to which reductionism imposes 'methodological caveats' restricting theory choice. He calls the attention of the anti-reductionists claiming that

the new-wave construal of the reductionist thesis is a prediction (...) [t]he evidence for this prediction is current developments in the relevant sciences and projected future developments of approaches now gaining steam' (1998, 140, emphasis in the original).

Given Bickle's failure, we should expand this anti-normative/antiregulative trend to the ontological conclusions as well. Likewise theory choice, ontological decisions are part of scientific practice itself. We should evaluate reductionist relations among theories taking 
seriously the scientific community evaluations like Patricia Churchland advises us to $\mathrm{do}^{2}$.

João Fonseca

Instituto de Filosofia da Linguagem Faculdade de Ciências Sociais e Humanas

Universidade Nova de Lisboa Avenida de Berna, 26-C, 1069-061 Lisboa, Portugal jfonseca.ifl@fcsh.unl.pt

References

Balzer, W., Moulines, C. U., and Sneed, 1987. An Architectonic for Science. Dordrecht: Reidel.

Bickle, J., 1998. Psychoneural Reduction: The New Wave. Cambridge, Mass.: The MIT Press.

Churchland, P. S., 1986. Neurophilosophy. Cambridge, Mass.: The MIT Press.

\footnotetext{
${ }^{2}$ In fact, Bickle has done such a move on his more recent book Philosophy and Neuroscience: A Ruthlessly Reductive Account (Kluwer Academic Publishers) (I must thank an anonymous referee for point out this to me).
} 